\title{
Candidemia neonatal, em hospital público do Mato Grosso do Sul
}

\author{
Neonatal candidemia in a public hospital \\ in Mato Grosso do Sul
}

\author{
Paula Cristhina Niz Xavier¹, Marilene Rodrigues Chang², Maína O. Nunes², \\ Durval B. Palhares ${ }^{3}$, Renato Andreotti e Silva ${ }^{4}$, Gisele Facholi Bonfim ${ }^{5}$ \\ e Nalvo F. Almeida Júnior ${ }^{6}$
}

\begin{abstract}
RESUMO
O objetivo de nosso estudo foi realizar tipagem molecular de 25 amostras clínicas de Candida spp, isoladas de crianças com candidemia, internadas na unidade de terapia intensiva neonatal de um Hospital Universitário entre 1998 a 2006. Dados demográficos e clínicos foram obtidos de prontuários para conhecimento dos aspectos clínicos e epidemiológicos. Identificação das leveduras foi feita por método convencional e a susceptibilidade antifúngica por método de microdiluição. 0 perfil genético foi determinado pela técnica de RAPD-PCR. Candida albicans (11; 44\%) e Candida parapsilosis $(10 ; 40 \%)$ foram as mais isoladas. Dezessete (68\%) dos recém-nascidos tinham peso inferior a 1.500g. Prematuridade (92\%), uso de cateter venoso central (100\%), foram as condições de risco mais associados. Dezenove (76\%) pacientes foram a óbito. Apenas uma cepa de Candida parapsilosis, mostrou ser sensível dose dependente ao fluconazol. Na análise molecular, foram observados 11 padrões genéticos distintos. Somente em dois casos foi observada relação epidemiológica, sugerindo mesma fonte de infecção.
\end{abstract}

Palavras-chaves: Candidemia. Candida. Neonatal. Tipagem molecular.

\section{ABSTRACT}

The aim of our study was to perform molecular typing on 25 clinical samples of Candida spp that were isolated from children with candidemia who were hospitalized in the neonatal intensive care unit of a university hospital between 1998 and 2006. Demographic and clinical data were obtained from the medical records to ascertain the clinical and epidemiological characteristics. Yeast identification was done using conventional methods and susceptibility to antifungals was assessed using a microdilution method. The genetic profile was determined using the RAPD-PCR technique. Candida albicans $(11 ; 44 \%)$ and Candida parapsilosis $(10 ; 40 \%)$ were the species most frequently isolated. Seventeen (68\%) of the newborns weighed less than 1,500g. Prematurity (92\%) and use of a central venous catheter (100\%) were the risk conditions with greatest association. Nineteen patients (76\%) died. Only one strain of Candida parapsilosis showed dose-dependent sensitivity to fluconazole. Molecular analysis showed 11 distinct genetic patterns. An epidemiological relationship was seen in only two cases, thus suggesting the same source of infection.

Key-words: Candidemia. Candida. Neonatal. Molecular typing.

As infecções sistêmicas causadas por leveduras são de difícil diagnóstico e normalmente estão correlacionadas com o isolamento do agente na hemocultura ${ }^{20}$. Infecções na corrente sanguínea (ICS) causada por Candida são cada vez mais freqüentes em Unidades de Terapia Intensiva Neonatal (UT'Ineo), sendo causa relativamente comum de sepse associada a alta mortalidade 3 . A razão deste aumento inclui vários fatores como: baixo peso, prematuridade, sistema imunológico deficitário, anomalias congênitas, uso de cefalosporinas de $3^{a}$ geração, técnicas agressivas de diagnóstico, uso de cateteres vasculares e prolongado período de internação ${ }^{12}{ }^{19}$.
O potencial patogênico das leveduras varia consideravelmente. Candida albicans é a mais freqüentemente isolada de amostras clínicas, mas entre os neonatos, Candida parapsilosis costuma ser mais prevalente $\mathrm{e}^{141920}$.

O presente estudo teve como objetivo: avaliação epidemiológica, caracterização molecular e determinação da susceptibilidade frente ao fluconazol, de Candida spp isoladas de pacientes com infecção na corrente sanguínea, internados na UTIneo do Hospital Universitário da Universidade Federal de Mato Grosso do Sul (HU-UFMS).

1. Faculdade Estácio de Sá de Campo Grande, Campo Grande, MS. 2. Departamento de Farmácia Bioquímica, Universidade Federal do Mato Grosso do Sul, Campo Grande, MS. 3. Departamento de Pediatria, Faculdade de Medicina Dr. Hélio Mandeta, Universidade Federal do Mato Grosso do Sul, Campo Grande, MS. 4. Laboratório de Biologia Molecular, Empresa Brasileira de Pesquisa Agropecuária Gado de Corte (EMBRAPA), Campo Grande, MS. 5. Curso de Pós-Graduação em Farmácia, Faculdade de Ciências Farmacêuticas, Universidade de São Paulo, São Paulo, SP. 6. Departamento de Computação e Estatística, Universidade Federal do Mato Grosso do Sul, Campo Grande, MS. Apoio Financeiro: FUNDECT/MS processo 41/100.115/2004.

Endereço para correspondência: Dra. Paula Cristhina Niz Xavier. R. Itacajá 06, c49, Vila Vilma, 79092-400 Campo Grande, MS.

Tel: $55679283-6620$

e-mail: pxavier@fes.br

Recebido para publicação em 19/12/2007

Aceito em 27/08/2008 
Além da importância do tema que é a sepse fúngica em neonatos, este estudo se faz relevante, porque é a primeira divulgação de dados sobre infecção na corrente sanguínea e análise molecular de Candida spp, no Estado de Mato Grosso do Sul.

\section{MATERIAL E MÉTODOS}

Este estudo foi do tipo descritivo retrospectivo, baseado em resultados positivos de hemocultura de pacientes internados na UTI neo entre dezembro de 1998 e outubro de 2006. Foi critério de inclusão, cepa de Candida spp estocada no Laboratório de Micologia do HU-UFMS. Os dados demográficos e clínicos foram consultados nos prontuários e posteriormente analisados no programa Epi info (CDC, versão 3.3.0.2), juntamente com os resultados laboratoriais.

A coleta para hemocultura foi feita de sangue periférico. A detecção das leveduras foi feita pelo sistema BACTEC@ (Becton Dickinson) e a identificação das espécies por meio de metodologia convencional: pesquisa do tubo germinativo, auxanograma e microcultivo ${ }^{11}$.

A susceptibilidade antifúngica frente ao fluconazol foi determinada pela técnica de microdiluição em caldo, de acordo com normas do National Committee for Clinical Laboratory Standards (NCCIS), $\mathrm{M} 27 \mathrm{~A} 2^{16}$. Como controle de qualidade foram utilizadas cepas ATCC 22019 de Candida parapsilosis e ATCC 6258 de Candida krusei.

Para a caracterização molecular, o DNA genômico foi extraído, purificado e a técnica de Random amplification of polymerase DNA-polymerase chain reaction (RAPD-PCR) realizada de acordo com Mannarelli \& Kurtzman ${ }^{9}$, utilizando um primer randômico, com sequiência: (5’ACGACCGACA 3') e DNA Ladder de 100 pares de base $(\mathrm{Pb})(105 \mathrm{ng} / \mu \mathrm{L}$ - Jena Bioscience - M214) como marcador de peso molecular. Os produtos amplificados foram separados em gel de agarose e corados com brometo de etídio. A interpretação dos padrões obtidos foi realizada por meio do programa PARS incluído no PHYLIP Package, versão 3.667.

Os conceitos de cepa endêmica e relacionada a surto de infecção seguem os critérios de Tenover ${ }^{21}$, sendo consideradas amostras epidemiologicamente relacionadas as cepas de uma mesma espécie cultivada de pacientes internados na mesma unidade em um curto espaço de tempo e com o mesmo perfil genético.

Considerações éticas. 0 estudo foi aprovado pelo Comitê de Ética e Pesquisa da Universidade Federal do Mato Grosso do Sul, conforme Parecer no ${ }^{4} 65$.

\section{RESULTADOS}

Dos 25 casos de candidemia que foram estudados, 23 eram prétermos, um recém-nascido de termo e um com idade gestacional (IG) indeterminada, 48\% dos recém-nascidos tinham IG menor que trinta semanas. Dezessete (68\%) neonatos tinham peso inferior a $1.500 \mathrm{~g}$ ao nascimento e menos de um mês de vida (média de 16,76 dias), na ocasião do diagnostico de candidemia. Outros dados demográficos e clínicos estão demonstrados na Tabela 1.

Tabela 1 - Características demográficas, clínicas e evolução de 25 recém-nascidos com candidemia internados no Hospital da Universidade Federal do Mato Grosso do Sul, 1998-2006.

\begin{tabular}{|c|c|c|c|c|c|c|c|c|c|}
\hline Caso & Data & Espécie & $\begin{array}{c}\text { Perfil } \\
\text { DNA }\end{array}$ & $\begin{array}{l}\text { Idade } \\
\text { (dias) }\end{array}$ & $\begin{array}{c}\text { Idade } \\
\text { gestacional } \\
\text { (semanas) }\end{array}$ & $\begin{array}{c}\text { Peso } \\
\text { (gramas) }\end{array}$ & Sexo & $\begin{array}{l}\text { Diagnóstico } \\
\text { clínico }\end{array}$ & Evolução \\
\hline 1 & $12 / 10 / 97$ & Candida parapsilosis & $\mathrm{B}$ & 5 & 31 & 1.500 & $\mathrm{~F}$ & PMT/má formação congênita & alta \\
\hline 2 & $27 / 03 / 98$ & Candida parapsilosis & G & 42 & não consta & 3.090 & M & sepse & óbito \\
\hline 3 & $08 / 08 / 98$ & Candida tropicalis & $\mathrm{D}$ & 6 & 32 & 2.800 & M & PMT/doença trato gastrintestinal & óbito \\
\hline 4 & $13 / 02 / 99$ & Candida parapsilosis & B & 50 & 28 & 1.030 & M & PMT/sepse & óbito \\
\hline 5 & $14 / 02 / 99$ & Candida albicans & A & 45 & 30 & 1.150 & M & PMT/pneumonia & óbito \\
\hline 6 & $07 / 03 / 00$ & Candida tropicalis & $\mathrm{D}$ & 41 & 30 & 1.030 & M & PMT/sepse & óbito \\
\hline 7 & $27 / 03 / 00$ & Candida albicans & $\mathrm{J}$ & 30 & 32 & 1.850 & M & $\mathrm{PMT} / \mathrm{sepse}$ & alta \\
\hline 8 & $11 / 10 / 00$ & Candida parapsilosis & B & 35 & 26 & 785 & $\mathrm{~F}$ & PMT/sepse & óbito \\
\hline 9 & $23 / 10 / 00$ & Candida parapsilosis & B & 16 & 26 & 1.050 & M & PMT/pneumonia./sepse & óbito \\
\hline 10 & $14 / 08 / 01$ & Candida tropicalis & $\mathrm{E}$ & 13 & 34 & 1.385 & M & PMT/pneumonia/sepse & óbito \\
\hline 11 & $26 / 12 / 01$ & Candida albicans & $\mathrm{C}$ & 28 & 29 & 1.280 & M & PMT & óbito \\
\hline 12 & $01 / 02 / 03$ & Candida albicans & A & 18 & 28 & 1.108 & M & PMT/sepse & aAlta \\
\hline 13 & $27 / 02 / 03$ & Candida glabrata & $\mathrm{H}$ & 66 & 31 & 1.185 & M & PMT/sepse & óbito \\
\hline 14 & $03 / 04 / 03$ & Candida parapsilosis & K & 13 & 36 & 1.740 & M & PMT/sepse & óbito \\
\hline 15 & 05/04/03 & Candida albicans & A & 21 & 40 & 3.360 & $\mathrm{~F}$ & sepse & alta \\
\hline 16 & $09 / 04 / 03$ & Candida parapsilosis & $\mathrm{F}$ & 12 & 36 & 3.190 & M & PMT/sepse & óbito \\
\hline 17 & $30 / 05 / 03$ & Candida albicans & A & 13 & 27 & 1.035 & $\mathrm{~F}$ & PMT/sepse & óbito \\
\hline 18 & $25 / 08 / 04$ & Candida albicans & A & 19 & 27 & 1.090 & $\mathrm{~F}$ & PMT/sepse & óbito \\
\hline 19 & $13 / 06 / 05$ & Candida parapsilosis & B & 10 & 28 & 875 & $\mathrm{~F}$ & PMT/sepse & óbito \\
\hline 20 & $06 / 01 / 06$ & Candida parapsilosis & I & 15 & 26 & 810 & $\mathrm{~F}$ & PMT/sepse & óbito \\
\hline 21 & $22 / 02 / 06$ & Candida albicans & A & 29 & 31 & 1.355 & $\mathrm{~F}$ & PMT/sepse & óbito \\
\hline 22 & 03/03/06 & Candida albicans & $\mathrm{C}$ & 28 & 32 & 1.500 & $\mathrm{~F}$ & PMT/sepse & alta \\
\hline 23 & $04 / 05 / 06$ & Candida albicans & A & 28 & 27 & 1.035 & $\mathrm{~F}$ & PMT/sepse & óbito \\
\hline 24 & $09 / 08 / 06$ & Candida parapsilosis & - & 16 & 30 & 1.405 & $\mathrm{~F}$ & PMT/incompatibilidade Rh & alta \\
\hline 25 & $04 / 12 / 06$ & Candida albicans & - & 16 & 26 & 1.410 & M & PMT/infecção neonatal & óbito \\
\hline
\end{tabular}

DNA: ácido desoxirribonucléico, F: feminino, M: masculino, PMT: prematuridade, idade gestacional < 37 semanas. 
Candida albicans foi a causa mais comum de candidemia (11; 44\%), seguida de Candida parapsilosis (10; 40\%), Candida tropicalis $(3 ; 12 \%)$, Candida glabrata $(1 ; 4 \%)$.

As condições de risco mais prevalentes entre os pacientes com infecção na corrente sanguínea foram uso de cateter venoso central e ventilação mecânica $(25 ; 100 \%)$, prematuridade $(23 ; 92 \%)$, tempo de internação superior a 15 dias $(20 ; 80 \%)$, uso de nutrição parenteral (23;92\%), sonda nasogástrica $(20 ; 80 \%)$, cefalosporinas de $3^{\text {a }}$ geração $(19 ; 76 \%)$, dreno $(7 ; 28 \%)$ e procedimento cirúrgico (14; 56\%). Em 4 casos, a mesma levedura foi isolada em cultura de ponta de cateter e da hemocultura do paciente.
Cerca de $80 \%$ dos pacientes foram tratados com antifúngicos, sendo que 12 (48\%) usaram anfotericina B, 16 (64\%) fluconazol e $9(36 \%)$, utilizaram ambos antifúngicos. Dezenove (76\%) crianças foram a óbito durante a hospitalização.

A Tabela 2 mostra o perfil de susceptibilidade in vitro de 22 isolados causadores de candidemia frente ao fluconazol. $\mathrm{Na}$ análise molecular, foram observados 11 padrões genéticos distintos. As Figuras 1 e 2 ilustram os diferentes perfis encontrados para as espécies Candida albicans e Candida parapsilosis.

Tabela 2 - Perfil de susceptibilidade in vitro de Candida spp isoladas de recém-nascidos com infecção em corrente sanguínea, internados na UTIneo do Hospital Universitário da Universidade Federal do Mato Grosso do Sul, frente ao fluconazol.

\begin{tabular}{|c|c|c|c|c|c|c|c|c|c|}
\hline \multirow[t]{2}{*}{ Espécie } & \multirow[t]{2}{*}{ Número } & \multirow[t]{2}{*}{ Porcentagem } & \multirow{2}{*}{$\begin{array}{c}\text { Variação } \\
\text { CIM }_{50} \\
(\mu \mathrm{g} / \mathrm{mL})\end{array}$} & \multicolumn{2}{|c|}{ Sensível } & \multicolumn{2}{|c|}{ SDD } & \multicolumn{2}{|c|}{ Resistente } \\
\hline & & & & $\mathrm{n}^{\mathrm{o}}$ & $\%$ & $\mathrm{n}^{0}$ & $\%$ & $\mathrm{n}^{\underline{0}}$ & $\%$ \\
\hline Candida albicans & 9 & 40,9 & $0,25-2$ & 9 & 100 & & - & - & - \\
\hline Candida parapsilosis & 9 & 40,9 & $0,5-32$ & 7 & 87,5 & 1 & 12,5 & - & - \\
\hline Candida tropicalis & 3 & 13,6 & $0,5-1$ & 3 & 100 & & - & - & - \\
\hline Candida glabrata & 1 & 4,5 & 8 & 1 & 100 & & - & - & - \\
\hline
\end{tabular}

$\mathrm{CIM}_{50}$ : concentração inibitória mínima, SDD: sensível dose dependente

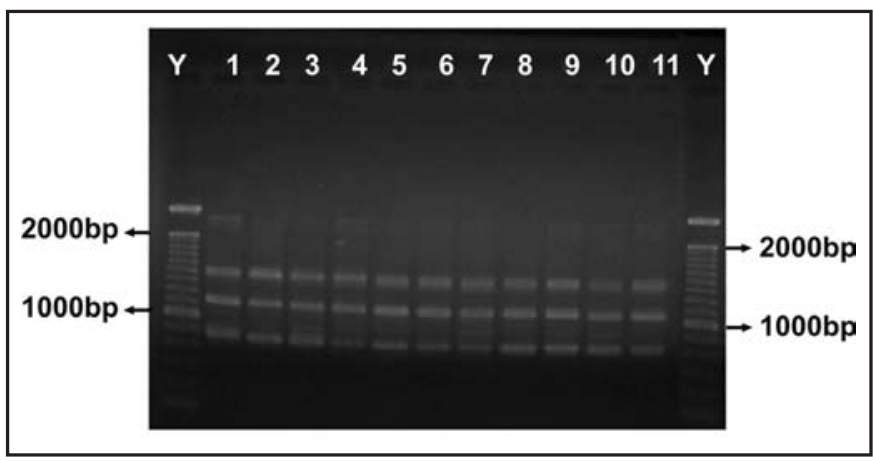

Linhas $\mathrm{Y}=$ marcador de peso molecular, Linha 1-Candida albicans ATCC 10231; L2 - 530H/99; L3713H5/01; L4-007H/03; L5- 486H3/03; L6- 727H3/03; L7 - 82H/04; L8- 003H/06; L9- 982H/06; L10- 340H/06; L11- 175H4/00.

Figura 1 - Eletroforese em gel de agarose após RAPD-PCR ilustrando diferentes padrões genéticos de Candida albicans, agentes de infecção em corrente sangüínea de neonatos, utilizando o Primer 5'd[ ACGACCGACA]- 3'.

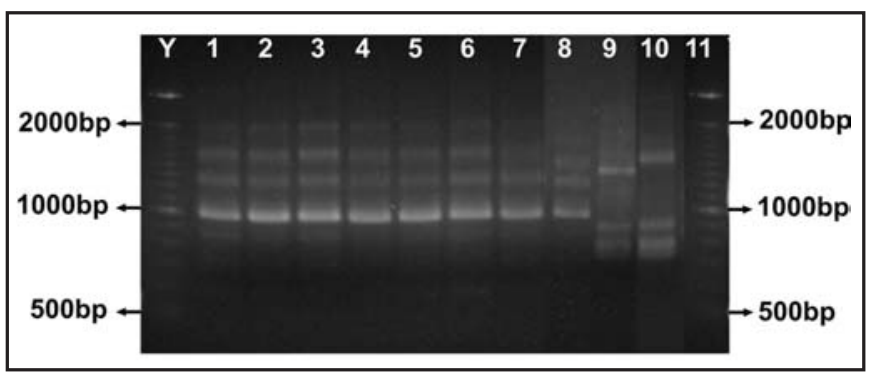

Linhas $\mathrm{Y}=$ marcador de peso molecular, Linha 1-Candida parapsilosis ATCC 22019; L2 $-005 \mathrm{H}_{10} / 00$ (B); $\mathrm{L}-990 \mathrm{H}_{10} / 00$ (B); $\mathrm{L}_{4}-709 \mathrm{H}_{8} / 06$ (B); L5-264H 11 /97 (B); L6- $434 \mathrm{H}_{2} / 99$ (B); L7- 120H/05 (B); L8 - 118H3/98 (G); L9- 703H/03 (K); L10- 736H/03 (F).

Figura 2 - Eletroforese em gel de agarose após RAPD-PCR ilustrando diferentes padrões genéticos de Candida parapsilosis, agentes de infecção em corrente sangüinea de neonatos, utilizando o Primer 5'd[ ACGACCGACA]- 3'. 


\section{DISCUSSÃo}

Nas últimas décadas, espécies de Candida têm emergido como patógenos comuns em recém nascidos de baixo peso, sendo considerada a quarta causa de infecções na corrente sanguínea ${ }^{20}$. Pacientes com candidemia apresentam síndrome séptica aguda que é indistinguível de bacteremia ${ }^{19}$.

A prematuridade associada a procedimentos invasivos como: cateter venoso central, ventilação mecânica, além de uso de nutrição parenteral, uso prévio de antibióticos, etc, podem favorecer a infecção por patógenos oportunistas como Candida $\mathrm{spp}^{12}{ }^{14}$. Neste estudo, isso pode ser constatado pelo isolamento do mesmo patógeno na cultura do cateter venoso central e do sangue do paciente.

A letalidade encontrada ( $76 \%$ ) neste estudo, foi superior a outros relatos ${ }^{613}$. Entretanto, fica difícil atribuir essa frequiência à infecção na corrente sanguínea pela levedura, porque as crianças freqüentemente tinham problemas respiratórios, gastrintestinais e outras complicações que poderiam também estar relacionada ao óbito.

Estudos de vigilância epidemiológica têm demonstrado que as espécies de Candida não albicans têm emergido como importantes agentes de fungemias ${ }^{618}$. Isso também pode ser observado na presente investigação, onde o número de Candida não albicans $\left(\mathrm{n}^{0}=14\right)$ foi superior ao de Candida albicans $\left(\mathrm{n}^{\mathrm{0}}=11\right)$. Entre as espécies de Candida não albicans, Candida parapsilosis foi a mais frequientemente isolada, similar ao encontrado por outros pesquisadores $^{38}$. Diferente do observado em estudos norteamericanos $^{910} 17$ em nossa instituição, Candida glabrata ainda apresenta baixa freqüência de isolamento nas hemoculturas. Vale ressaltar que vigilância contínua se faz necessária para monitorar a emergência e disseminação de patógenos oportunistas como Candida em pacientes de alto risco, como os neonatos.

$\mathrm{Na}$ análise das concentrações inibitórias mínimas encontradas, observou-se que todas as espécies de Candida isoladas (com exceção de uma Candida parapsilosis que foi considerada sensível dose dependente), foram sensíveis in vitro ao fluconazol, similar a estudos com dados multicêntricos ${ }^{910} 17$. Esse antifúngico tem sido utilizado de forma profilática em nosso hospital assim como em outras instituições hospitalares ${ }^{8}$. Por outro lado, segundo Aghai $\operatorname{cols}^{1}$, o uso profilático do fluconazol deve ser utilizado com cautela em recém-nascido de baixo peso.

Recentemente, a utilização de técnicas de biologia molecular tem sido considerada uma poderosa ferramenta para investigação de surtos de infecção hospitalar ${ }^{15}{ }^{18}$. Em relação aos padrões genéticos obtidos entre as cepas estudadas, constatou-se predomínio do clone A, de Candida albicans e do clone B, de Candida parapsilosis, com cepas endêmicas presentes na UTIIneo que apareceram e desapareceram durante o período estudado, sem, entretanto provocar grandes surtos. Acredita-se que pode ter havido transmissão cruzadas entre o caso 8 e caso 9, por estarem epidemiologicamente relacionadas. Estudos mais amplos são necessários para melhor entendimento da epidemiologia molecular das candidemias.

\section{AGRADECIMENTOS}

ADr ${ }^{\text {a }}$ Flávia Emi Matsumoto do Instituto de Ciências Biomédicas da Universidade de São Paulo (ICB-USP), pelo treinamento na técnica de extração de DNA e RAPD-PCR. A Jacqueline Cavalcante Barros do Laboratório de Biologia Molecular da EMBRAPA Gado de Corte, pelo apoio técnico no laboratório no qual a técnica de PCR foi realizada.

\section{REFERÊNCIAS}

1. Agarwal J, Bansal S, Malik GK, Jain A. Trends in neonatal septicemia: emergence of non-albicans. Indian Pediatric 7: 712-715, 2004

2. Aghai ZH, Mudduluru M, Nakhla TA, Amendolia B, Longo D, Kemble N, Kaki S, Sutsko R, Saslow JG, Stahl GE. Fluconazole prophylaxis in extremely low birth weight infants: association with cholestasis. Journal of Perinatology 26: 550-555, 2006.

3. Benjamin Jr DK, Stoll BJ, Fanaroff AA, Mcdonald SA, Oh W, Higgins RD, Duara S, Poole K, Laptook A, Goldberg R. Neonatal candidiasis among extremely low birth weight infants: risk factors, mortality rates, and neurodevelopmental outcomes at 18 to 22 months. Pediatrics 117:84-92, 2006.

4. Cheng MF, Yang YL, Yao TJ, Lin CY, Liu JS, Tang RB, Yu KW, Fan YH, Hsieh KS, Ho M, Lo HJ. Risk factors for fatal candidemia caused by Candida albicans and non-albicas Candida. BioMed Central Infectious Diseases 5: 1-5, 2005.

5. Colombo AL, Guimarães T. Epidemiologia das infecções hematogênicas por Candida spp. Revista da Sociedade Brasileira de Medicina Tropical 36: 599-607, 2003.

6. Diekema DJ, Messer SA, Brueggemann AB, Coffman SL, Doern GV, Herwaldt LA, Pfaller MA. Epidemiology of candidemia: 3-year results from the emerging infections and the epidemiology of Iowa organisms study. Journal of Clinical Microbiology 40: 1298-1302, 2002.

7. Felsenstein J. Inferring Phylogenies underland, MA: Sinauer Associates, 2004.

8. Fridkin SK, Kaufman D, Edwards JR, Shetty S, Horan T, National Nosocomial Infection Surveillance System Hospitals. Changing incidence of candida Bloodstream infections among NICU patients the United State: 1995-2004. Pediatrics 117: 1680-1687, 2006.

9. Godoy P, Tiraboschi IN, Severo LC, Bustamante B, Calvo B, Da Matta DA, Colombo AL. Species distribuition and antifungal susceptibility profile of Candida spp. bloodstream isolates from Latin America hospitals. Memória do Instituto Oswaldo Cruz 98: 401-405, 2003.

10. Hajjeh RA, Sofair AN, Harrison LH, Lyon MG, Arthington-Skaggs BA, Mirza SA Phelan M, Morgan J, Lee-Yang W, Ciblak MA, Benjamin LE, Sanza LT, Yeo SHSF Brandit ME, Warnock DW. Incidence of bloodstream infections due to Candida species and in vitro susceptibilities of isolates collected from 1998 to 2000 in a population-based active surveillance program. Journal of Clinical Microbiology 42:1519-1527, 2004.

11. Lacaz CS, Porto E, Martins JEC, Heins-Vaccari E, Melo NT. Tratado de Micologia Médica. $9^{a}$ edição, Editora Sarvier, São Paulo, 2002.

12. Lupetti A, Tavanti A, Davini P, Ghelardi E, Corsini V, Merusi I, Boldrini A, Campa M, Senesi S. Horizontal transmisson of Candida parapsilosis Candidemia in a neonatal intensive care unit. Journal of Clinical Microbiology 40: 2363-2369, 2002 .

13. Mannarelli BM, Kurtzman CP. Rapid identification of Candida albicans and other human pathogenic yeasts by using short oligonucleotidess in a PCR. Journal of Clinical Microbiology 36:1634-1641, 1998.

14. Matsumoto FE, Gandra RF, Ruiz LS, Auler ME, Marques SAV, Pires MFC, Gambale W, Paula, CR. Yeasts isolated from blood and catheter in children from a public hospital of São Paulo, Brazil. Mycopathologia 154: 63-69, 2001.

15. Mirhend H, Makimura K, Khoramizadreza M, Yamaguichi H. A one-enzyme PCRRFLP assay for identification of six medically important Candida species. Journal Medicine Mycology 47: 225-229, 2006. 
16. National committee for clinical laboratory standards. Reference method for broth microdilution antifungal susceptibility testing of yeasts. Approved standard M27A2, National Committee for Clinical Laboratory Standards, Wayne, PA, 2002.

17. Pfaller AM, Diekema DJ. Role of sentinel surveillance of candidemia: treds in species distribution and antifungal susceptibility Journal of Clinical Microbiology 40: 3551-3557, 2002.

18. Roilides E, Farmaki E, Evdoridou, Dotis J, Hatziioannidis E, Tsivitanidou M, Bibashi E, Filioti I, Sofianou D, Gil-Lamaignere C, Mueller FM, Kremenopoulos G. Neonatal candidiasis: analysis of epidemiology, drug susceptibility, and moleular typing of causative isolates. European Journal of Clinical Microbiology and Infectious Diseases 23: 745-750, 2004.

19. Saiman L. Neonatal Candidiasis. Clinical Microbiology Newsletter 20: 149-155, 1998.

20. Sandven P. Epidemiology of candidemia. Revista Iberoamericana de Micologia 17: 73-81, 2000.

21. Tenover FC, Arbeit RD, Goering RV. Interpreting chromosomal DNA restriction patterns produced by pulsed-field gel electrophoresis: criteria for bacterial strain typing. Journal of Clinical Microbiology 33: 2233-2239, 1995. 